OPEN ACCESS

Edited by: Yassine Taoufik, Université Paris-Sud, France

Reviewed by: Reinhard Reuß, BKH Bayreuth, Germany Bruno Stankoff,

University Pierre et Marie Curie, France; APHP-Hôpital Tenon, France

*Correspondence: Eli Hatchwell, Population Diagnostics UK, Inc., Begbroke Science Park, Begbroke Hill, Woodstock Road, Begbroke, Oxfordshire OX5 1PF, UK elihatchwel/@ populationdiagnostics.com

Specialty section: This article was submitted to Multiple Sclerosis and Neuroimmunology, a section of the journal Frontiers in Immunology

Received: 04 December 2014 Accepted: 20 April 2015 Published: 11 May 2015

Citation: Hatchwell E (2015) Is there a (host) genetic predisposition to progressive multifocal leukoencephalopathy? Front. Immunol. 6:216. doi: 10.3389/fimmu.2015.00216

\section{Is there a (host) genetic predisposition to progressive multifocal leukoencephalopathy?}

\author{
Eli Hatchwell * \\ Population Diagnostics UK, Inc., Begbroke Science Park, Begbroke, Oxfordshire, UK
}

Progressive multifocal leukoencephalopathy $(\mathrm{PML})$ has been described in association with a variety of predisposing risk factors, including HIV/AIDS, lymphoproliferative disorders and, most recently, treatment with a range of biologics, most notably natalizumab in multiple sclerosis (MS) (1). However, while these underlying disorders appear to be (usually) necessary, they are not sufficient to predict the development of PML, since only a small fraction of such individuals will succumb, raising the question of whether host genetic factors must also play a role. Evidence is mounting that this is, indeed, the case but more work needs to be done to fully delineate these underlying genetic factors. Finally, while it is possible that an underlying genetic susceptibility for PML in general will be uncovered in the future, the current evidence argues for a collection of multiple, individually rare underlying susceptibilities (with one predominant single genetic susceptibility in each individual), as described in this review.

Keywords: progressive multifocal leukoencephalopathy, host genetic factors, primary immunodeficiencies, PML predisposition, natalizumab

\section{Introduction}

Progressive multifocal leukoencephalopathy (PML) is a severe, potentially fatal, CNS infection. It is "caused" by reactivation of the JC virus (JCV). However, while JCV is present at very high rates in the general population, PML remains a rare disorder, albeit an important one because of the clinical sequelae, and the recently demonstrated association with a variety of useful therapies, in particular natalizumab in multiple sclerosis (MS). A number of risk factors for PML have been described but these are better viewed as necessary but not sufficient. The majority of individuals with these risk factors do not develop PML and so the obvious question is "In addition to the well-known risk factors for PML, are there host genetic factors that also play a role?"

\section{"Traditional” Risk Factors for PML}

This is a topic that has been well reviewed in the literature [for a recent comprehensive discussion, see Ref. (1)]. In brief, while rare individuals have been reported, in whom no obvious risk factors are present (2), the majority of individuals have a history of profound cellular immunosuppression, whether associated with systemic disorders, such as HIV, lymphoproliferative disease, rheumatological disorders (SLE, for example), or else treatment with immunosuppressive or immunomodulatory therapies. Historically, PML (first described in the 1950s) was known mainly as a disease associated with lymphoproliferative disorders. Knowledge of PML increased with the advent of AIDS, as 4-5\% of patients developed PML. In recent years, PML has come to prominence in the context of the 
drug natalizumab, an effective biologic used in the treatment of MS. PML has also been described in association with other biologics [reviewed in Ref. (1)] and, most recently, with the use of drugs containing fumarates (used in the treatment of psoriasis and also the MS drug Tecfidera) (3).

The important point is that while these risk factors are highly relevant, they do not, on their own, predict who will develop PML, since the vast majority of individuals with these risk factors will not develop the disorder. Clearly, other factors need to be considered and there is growing evidence for the role of host genetic factors in susceptibility to PML.

Finally, the rare description of individuals that have developed PML in the complete absence of any known "traditional" risk factor argues very strongly for the presence of host genetic factors that result in an inability to effectively deal with JCV.

\section{Precedents for Genetic Predisposition to Specific Infectious Disease}

Many factors/conditions are known to predispose to infectious disease in general. However, what is of greater interest is the existence of genetic syndromes that appear to predispose to severe infection with very specific infectious agents. A classical example is X-linked lymphoproliferative syndrome (XLP), a primary immunodeficiency disorder (PID) characterized by severe immune dysregulation often after viral infection, typically with Epstein-Barr virus (EBV). XLP is present in two forms, each associated with mutations in a different gene: XLP1 is associated with SH2D1A mutations, while XLP2 is associated with XIAP mutations. Both genes are located in Xq25 (4). More recently, Herpes simplex virus encephalitis (HSE), a rare complication of infection with HSV-1 has been shown to be associated with deficiency of TLR3 at 4q35.1 (5).

\section{Known/Suspected Genetic Predispositions for PML}

The association of PML with rare immune deficiency syndromes is suggested by a number of case reports, some of which predated the ability to confirm the clinical diagnosis of the underlying disorder by specific gene testing.

Table 1 summarizes specific PIDs in which PML has been reported, along with estimates for the numbers of distinct PML cases observed.

\section{Wiskott-Aldrich Syndrome}

Wiskott-Aldrich syndrome (WAS) is an X-linked recessive immunodeficiency, caused by mutations in the WAS gene at $\mathrm{Xp11.23}$. A similar disorder, WAS2, is caused by recessive mutations in WIPF11, at $2 \mathrm{q} 31.1$. There are five reports of PML in association with clinically diagnosed WAS. It is unclear why WAS is the most common PID described in association with PML. This may simply be due to ascertainment bias, given that the small number of cases reported early on may have alerted clinicians to specifically test for this condition. Alternatively, this may simply be a sampling issue, given the rarity of PML.
TABLE 1 | Summary of specific PIDs reported in association with PML.

\begin{tabular}{llcc}
\hline Disorder predisposing to PML & Gene & $\begin{array}{c}\text { Number } \\
\text { PML cases }\end{array}$ & Reference \\
\hline Wiskott-Aldrich Syndrome & WAS, WIPF11 & 5 & $(6-10)$ \\
$\begin{array}{l}\text { Severe combined } \\
\text { immunodeficiency syndrome }\end{array}$ & Various & 2 & $(11,12)$ \\
X-linked hyper-lgM Syndrome & CD40LG & 3 & $(13-15)$ \\
Hyper IgE Syndrome & DOCK8, STAT3, & 2 & $(16,17)$ \\
ICF syndrome & TYK2, STK3 & 1 & $(18)$ \\
Adenosine deaminase deficiency & ADA & 1 & $(19)$ \\
Idiopathic CD4 deficiency & RAG1, MST1, & 1 & $(20)$ \\
X-linked agammaglobulinemia & BTK & 1 & $(21)$ \\
$\begin{array}{l}\text { Purine nucleoside phosphorylase } \\
\text { deficiency }\end{array}$ & PNP & 1 & $(22)$ \\
STAT1 gain of function & & 1 & $(23)$ \\
\hline
\end{tabular}

Note that references refer to $P M L$ case reports but genes listed refer to known causes of the syndrome, even if some specific genes have not yet been implicated in PML per se.

\section{Severe Combined Immunodeficiency Syndrome}

Severe combined immunodeficiency syndrome (SCID) refers to a genetically and clinically heterogeneous group of disorders with defective cellular and humoral immune function. There have been at least two reports of PML in association with SCID.

\section{X-Linked Hyper-IgM Syndrome}

$\mathrm{X}$-Linked Hyper-IgM Syndrome is caused by mutations in the CD40LG (aka TNFSF5) gene at Xq26.3. It is a rare immunodeficiency characterized by normal/elevated IgM levels but markedly decreased $\operatorname{IgG}$, IgA, and IgE resulting in a profound susceptibility to bacterial infections and an increased susceptibility to opportunistic infections. It has been described in four patients reported as having PML.

\section{Hyper-IgE Syndrome}

Hyper-IgE recurrent infection syndrome is a PID characterized by chronic eczema, recurrent staphylococcal infections, increased serum IgE, and eosinophilia. It may be associated with dominant mutations in STAT3 at 17q21.2, recessive mutations in DOCK8 at $9 \mathrm{p} 24.3$, or recessive mutations in TYK2 at 19p13.2 (only one case reported). Most recently, a patient with PML was reported with a $>500 \mathrm{~kb}$ homozygous deletion encompassing a number of genes in 9p24.3, with DOCK8 the strongest candidate for PML susceptibility. Finally, there was also a report of a patient with PML associated with DOCK8 mutation in 2010.

\section{ICF syndrome}

Immunodeficiency, Centromeric instability, and Facial dysmorphism (ICF) syndrome is a rare autosomal recessive syndrome associated with mutations in DNMT3B at 20q11.21. One PML case has been reported in a patient with ICF.

\section{Adenosine Deaminase Deficiency}

Inherited Adenosine Deaminase (ADA) deficiency causes a spectrum of disease, from SCID, presenting in infancy and usually 
resulting in early death, to a later onset at age 6-24 months, and, finally, a small percentage of cases with a "later" onset, presenting from 4 years of age to adulthood. The disorder is associated with mutations in ADA at 20q13.12. There has been one case of PML reported in the context of ADA deficiency.

\section{Idiopathic CD4 Deficiency}

Idiopathic CD4 lymphocytopenia (ICL) is a syndrome described in patients with low counts of CD4 cells and no other causes for immunosuppression. A few cases of PML have been described in association with this entity. Mutations in a number of genes have been reported in association with ICL, including RAG1 at 11p.12, MST1 at 3p21.31, LCK at 1p35.1, and ITK at 5q33.3. One case of PML has been reported in association with ICL.

\section{X-Linked Agammaglobulinemia}

$\mathrm{X}$-linked agammaglobulinemia (XLA) is caused by mutations in the BTK gene at Xq22.1. It results in an immunodeficiency characterized by failure to produce mature B lymphocytes and is associated with a failure of Ig heavy chain rearrangement. One case of PML has been reported in association with XLA.

\section{Purine Nucleoside Phosphorylase Deficiency}

Purine nucleoside phosphorylase (PNP) deficiency is a rare autosomal recessive immunodeficiency disorder characterized mainly by decreased $\mathrm{T}$ cell function. It is caused by mutations in the PNP gene at $14 \mathrm{q} 11.2$ One case has been reported with PML.

\section{STAT1 Gain of Function}

Mutations in STAT1 (2q32.2) have been implicated in a number of disorders, including susceptibility to a variety of infectious agents, depending on which of four main subtypes of STAT1 is present. In one study describing the association between STAT gain of function mutations and disseminated coccidioidomycosis and histoplasmosis, one of five patients was reported with PML.

\section{Challenges of Genetic Studies into PML}

While the reports of rare individuals with PML in association with a confirmed PID immediately suggest a set of candidate genes that should be routinely searched for in PML patients, even when no such obvious (clinical) diagnosis has been made, it is possible that a more subtle and specific immunodeficiency will explain the majority of cases of PML, namely an immunodeficiency that is not clinically recognizable except in the context of JCV (i.e., one that does not result in other opportunistic infections, as is the case for XLP and specific susceptibility to EBV, or has other obvious associated clinical features that would lead to a diagnosis of a known PID). However, it is important to note that precedents do exist for the role of Mendelian genes, initially identified in severe disorders, being relevant to general populations in whom the severe phenotype is not present. An example of this has recently been demonstrated in late-onset Alzheimer's disease (LOAD), with the finding that rare variants in PSEN1, PSEN2, and APP increase the risk of Alzheimer's in LOAD families.
While a candidate gene list presents itself, there do remain significant challenges for the genetic dissection of PML predisposition, not only because the examples of PIDs described in association with PML are likely to account for only a small subset of the overall susceptibility to this disorder but because of more general considerations.

Progressive multifocal leukoencephalopathy remains an uncommon disorder, whose geographical distribution is wide. Very few specialists/centers have seen more than a handful of cases and collecting samples from sufficient numbers of cases has been challenging. Furthermore, based on the number of distinct PIDs in which PML has been reported in at least some cases, it is likely that the number of genes that might predispose to PML is significant, so that the unbiased identification of rare, causal variants will prove very difficult using modern whole genome or exome sequencing methods, for example, since the degree of background variation is simply too great to achieve statistical significance. The challenge of using next generation sequencing approaches is exacerbated by the possibility that some mutations may be dominantly acting (i.e., heterozygotes have the genetic susceptibility), a class of variants for which inferring causality is much more challenging than recessive disorders. Furthermore, the usual "tricks" employed to find significant causal variants in human genetic disorders either via traditional approaches or using NGS cannot be used because:

There are no:

1. families with Mendelian inheritance of PML (useful for traditional linkage analysis and/or segregation analysis of variants in suspected candidate genes), since, even were a dominant mutation to be segregating, it is highly unlikely that all carriers would also have the other, requisite, predispositions (underlying cellular immune suppression of whatever cause, for example);

2. obvious examples of PML arising as a result of a de novo mutation [in some childhood syndromes, such as severe intellectual disability (where parents are phenotypically normal), searches for de novo mutations have been fruitful];

3. other clinical/phenotypic clues that may aid in delineation of more specific subsets, for which correlations with underlying genetics may be useful (as occurs frequently in ID, wherein many ID syndromes are associated with specific physical manifestations that permit more specific disease delineation).

Discovery of the set of variants that underlie predisposition to PML is likely to depend on a combination of

1. consideration of strong candidate genes, in addition to those linked to already described PIDs. However, this is still a large class, based on the high number of genetically distinct conditions that are associated with immune suppression;

2. unbiased, genome-wide searches for causal variants, coupled with intelligent filtering of variants, based on known PIDs along with gene function as related to the immune system. However, such genome-wide searches for novel variants underlying PML development will not be straightforward;

3. the use of larger cohorts of PML subjects than has hitherto been possible. 


\section{Potential Benefits of Uncovering Genetic PML-Susceptibility Variants}

In the short term, the ability to more accurately predict who is at high risk of developing PML will be of enormous benefit in the context of drug treatment with compounds (mostly biologics) that are highly effective in their disease context (natalizumab in MS, for example) but carry a small risk of a devastating disorder. The hope is that patients in whom a course of treatment is being planned with such a drug would undergo initial, companion diagnostic testing, in order to effectively exclude those that were at high risk of PML, in the process reassuring those with negative tests about their dramatically reduced risk of developing PML.

Currently, the only approach in this direction is based on testing for the presence of JCV, since JCV is an absolute pre-requisite for PML development (24). However, because JCV is present in a large percentage of the normal population, this approach effectively excludes a significant proportion of patients from a treatment that is highly effective. In fact, it is possible that testing for JCV virus will not achieve its stated aim and patients will elect to accept the risk of PML, in return for improved quality of life on drug treatment (e.g., natalizumab in MS).

\section{Conclusion}

There appear to be three major criteria that must be fulfilled before PML occurs:

\section{References}

1. Calabrese LH, Molloy E, Berger J. Sorting out the risks in progressive multifocal leukoencephalopathy. Nat Rev Rheumatol (2015) 11(2):119-23. doi:10.1038/ nrrheum.2014.167

2. Gheuens S, Pierone G, Peeters P, Koralnik IJ. Progressive multifocal leukoencephalopathy in individuals with minimal or occult immunosuppression. J Neurol Neurosurg Psychiatry (2010) 81:247-54. doi:10.1136/jnnp.2009.187666

3. Sweetser MT, Dawson KT, Bozic C. Manufacturer's response to case reports of PML. N Engl J Med (2013) 368:1659-61. doi:10.1056/NEJMc1300283

4. Veillette A, Perez-Quintero LA, Latour S. X-linked lymphoproliferative syndromes and related autosomal recessive disorders. Curr Opin Allergy Clin Immunol (2013) 13:614-22. doi:10.1097/ACI.0000000000000008

5. Lim HK, Seppanen M, Hautala T, Ciancanelli MJ, Itan Y, Lafaille FG, et al. TLR3 deficiency in herpes simplex encephalitis: high allelic heterogeneity and recurrence risk. Neurology (2014) 83:1888-97. doi:10.1212/WNL. 0000000000000999

6. Katz DA, Berger JR, Hamilton B, Major EO, Post MJ. Progressive multifocal leukoencephalopathy complicating Wiskott-Aldrich syndrome. Report of a case and review of the literature of progressive multifocal leukoencephalopathy with other inherited immunodeficiency states. Arch Neurol (1994) 51:422-6. doi:10.1001/archneur.1994.00540160128016

7. Matsushima T, Nakamura K, Oka T, Tachikawa N, Sata T, Murayama S, et al. Unusual MRI and pathologic findings of progressive multifocal leukoencephalopathy complicating adult Wiskott-Aldrich syndrome. Neurology (1997) 48:279-82. doi:10.1212/WNL.48.1.279

8. Downes SM, Black GC, Hyman N, Simmonds M, Morris J, Barton C. Visual loss due to progressive multifocal leukoencephalopathy in a congenital immunodeficiency disorder. Arch Ophthalmol (2001) 119:1376-8. doi:10.1001/archopht. 119.9.1376

9. Otsubo K, Kanegane H, Nomura K, Miyawaki T, Ishizawa S. Atypical lymphoproliferative disorder in a patient with X-linked thrombocytopenia. Pediatr Blood Cancer (2008) 51:443-4. doi:10.1002/pbc.21644

10. Yasuda $Y$, Yabe $H$, Inoue $H$, Shimizu T, Yabe M, Yogo $Y$, et al. Progressive multifocal leukoencephalopathy after allogeneic bone marrow transplantation
1. JCV is present;

2. some form of cellular immune suppression is present, whether due to underlying systemic disease (such as hematological malignancy) or treatment with immunosuppressives (especially biologics, such as natalizumab);

3. host (genetic) susceptibility to develop PML;

For those rare individuals in whom no specific immune suppression is present in the medical history, there are two probably only essential factors:

1. JCV is present;

2. host (genetic) susceptibility to develop PML.

While there has been good progress in unraveling the epidemiology of PML, there is now some urgency to uncover as many of the underlying genetic variants as possible, primarily to create an effective and specific screening test that will determine, as definitively as possible, which patients should not be treated with a given therapeutic (MS, for example, when natalizumab is being considered) and which patients can safely (with dramatically reduced risk) be treated.

Finally, an improved understanding of the etiology of PML may, in the longer term, provide insights that will lead to therapies for individuals in whom PML has arisen as a result of their underlying disease, rather than a chosen treatment (e.g., HIV/AIDS and lymphoproliferative disorders).

for Wiskott-Aldrich syndrome. Pediatr Int (2008) 50:238-40. doi:10.1111/j. 1442-200X.2008.02549.x

11. Bledsoe T. Progressive multifocal leukoencephalopathy in a child with severe combined immunodeficiency. N Engl J Med (1978) 299:257-8. doi:10.1056/ NEJM197808032990517

12. Misbah SA, Spickett GP, Zeman A, Esiri MM, Wallington TB, Kurtz JB, et al. Progressive multifocal leucoencephalopathy, sclerosing cholangitis, bronchiectasis and disseminated warts in a patient with primary combined immune deficiency. J Clin Pathol (1992) 45:624-7. doi:10.1136/jcp.45.7.624

13. Suzuki H, Takahashi Y, Miyajima H. Progressive multifocal leukoencephalopathy complicating X-linked hyper-IgM syndrome in an adult. Intern Med (2006) 45:1187-8. doi:10.2169/internalmedicine.45.6023

14. Aschermann Z, Gomori E, Kovacs GG, Pal E, Simon G, Komoly S, et al. X-linked hyper-IgM syndrome associated with a rapid course of multifocal leukoencephalopathy. Arch Neurol (2007) 64:273-6. doi:10.1001/archneur.64.2.273

15. Pal E, Aschermann Z, Gomori E, Kovacs GG, Simon G, Marodi L, et al. [Progressive multifocal leukoencephalopathy]. Ideggyogy Sz (2007) 60:263-8.

16. Dellepiane RM, Engelhardt K, Pfeifer D, Beilis L, Raimondi M, Pavesi P, et al. Hyper IgE syndrome: fatal progressive multifocal leukoencephalopathy in an Italian child with DOCK8 mutation. Meeting of the European Society for Immunodeficiencies. Istanbul (2010).

17. G Day-Williams A, Sun C, Jelcic I, McLaughlin H, Harris T, Martin R, et al. Whole genome sequencing reveals a chromosome 9p deletion causing DOCK8 deficiency in an adult diagnosed with Hyper IgE syndrome who developed progressive multifocal leukoencephalopathy. J Clin Immunol (2015) 35:92-6. doi:10.1007/s10875-014-0114-4

18. Colucci M, Cocito L, Capello E, Mancardi GL, Serrati C, Cinque P, et al. Progressive multifocal leukoencephalopathy in an adult patient with ICF syndrome. J Neurol Sci (2004) 217:107-10. doi:10.1016/j.jns.2003.08.009

19. Jochum W, Weber T, Frye S, Hunsmann G, Luke W, Aguzzi A. Detection of JC virus by anti-VP1 immunohistochemistry in brains with progressive multifocal leukoencephalopathy. Acta Neuropathol (1997) 94:226-31. doi:10. 1007/s004010050697

20. Haider S, Nafziger D, Gutierrez JA, Brar I, Mateo N, Fogle J. Progressive multifocal leukoencephalopathy and idiopathic CD4+lymphocytopenia: a case 
report and review of reported cases. Clin Infect Dis (2000) 31:E20-2. doi:10. $1086 / 318120$

21. Teramoto T, Kaneko H, Funato M, Sawa H, Nagashima K, Hirose Y, et al. Progressive multifocal leukoencephalopathy in a patient with X-linked agammaglobulinemia. Scand J Infect Dis (2003) 35:909-10. doi:10.1080/ 00365540310016673

22. Parvaneh N, Ashrafi MR, Yeganeh M, Pouladi N, Sayarifar F, Parvaneh L. Progressive multifocal leukoencephalopathy in purine nucleoside phosphorylase deficiency. Brain Dev (2007) 29:124-6. doi:10.1016/j.braindev.2006.07.008

23. Sampaio EP, Hsu AP, Pechacek J, Bax HI, Dias DL, Paulson ML, et al. Signal transducer and activator of transcription 1 (STAT1) gain-of-function mutations and disseminated coccidioidomycosis and histoplasmosis. J Allergy Clin Immunol (2013) 131:1624-34. doi:10.1016/j.jaci.2013.01.052
24. Lee P, Plavina T, Castro A, Berman M, Jaiswal D, Rivas S, et al. A secondgeneration ELISA (STRATIFY JCV DxSelect) for detection of JC virus antibodies in human serum and plasma to support progressive multifocal leukoencephalopathy risk stratification. J Clin Virol (2013) 57:141-6. doi:10. 1016/j.jcv.2013.02.002

Conflict of Interest Statement: Eli Hatchwell is an employee of Population Diagnostics, Inc., UK.

Copyright (c) 2015 Hatchwell. This is an open-access article distributed under the terms of the Creative Commons Attribution License (CC BY). The use, distribution or reproduction in other forums is permitted, provided the original author(s) or licensor are credited and that the original publication in this journal is cited, in accordance with accepted academic practice. No use, distribution or reproduction is permitted which does not comply with these terms. 